\title{
Fisetin inhibits the growth and migration in the A549 human lung cancer cell line via the ERK1/2 pathway
}

\author{
JUNJIAN WANG and SHAOXIANG HUANG
}

\author{
Department of Respiratory Medicine, Tianjin 5th Central Hospital, Tianjin 300450, P.R. China
}

Received February 15, 2016; Accepted February 2, 2017

DOI: $10.3892 /$ etm.2017.5666

\begin{abstract}
Lung cancer is the most prevalent malignant tumor type in the developed world and the discovery of novel anti-tumor drugs is a research hotspot. Fisetin, a naturally occurring flavonoid, has been reported to have anti-cancer effects in multiple tumor types. The present study found that fisetin inhibited the growth and migration of non-small cell lung cancer in vitro. MTT, wound-healing, cell-matrix adhesion and Transwell assays were performed and demonstrated that fisetin suppressed proliferation, migration, adhesion and invasion, respectively. Flow cytometric analysis indicated that fisetin induced apoptosis in the A549 cell line by decreasing the expression of c-myc, cyclin-D1, cyclooxygenase-2, B cell lymphoma-2, CXC chemokine receptor type 4, cluster of differentiation 44 and metalloproteinase-2/9, increasing the expression of cyclin dependent kinase inhibitor (CDKN) $1 \mathrm{~A} / \mathrm{B}, \mathrm{CDKN} 2 \mathrm{D}$ and E-cadherin and increasing the activity of caspase-3/9 via targeting the extracellular signal-regulated kinase signaling pathway. The results provided comprehensive evidence for the anti-tumor effects of fisetin in non-small cell lung cancer in vitro, which may provide a novel approach for clinical treatment.
\end{abstract}

\section{Introduction}

Lung cancer is the most common tumor type in the developed world and in 2012 it was the most commonly diagnosed cancer (1.82 million) and the most common cause of cancer mortality (1.6 million) worldwide $(1,2)$. Lung cancer is classified into two major types: Small-cell lung cancer (SCLC) and non-small cell lung cancer (NSCLC). Most lung cancers are NSCLCs, which account for nearly $90 \%$ of cases (2). At present, surgical resection and chemotherapy are the major means of treating NSCLC. Over the last few decades, significant advances

Correspondence to: Dr Shaoxiang Huang, Department of Respiratory Medicine, Tianjin 5th Central Hospital, 41 Zhejiang Road, Tianjin 300450, P.R. China

E-mail: shaoxiang_huang@sina.com

Key words: fisetin, non-small cell lung cancer, growth, migration, extracellular signal-regulated kinase in diagnostic and therapeutic approaches have been made. However, $>80 \%$ of NSCLC patients cannot be treated with surgery due to the high prevalence of metastasis (3). According to statistics, the 5-year survival rate after lung cancer diagnosis is only $17.7 \%$ (4). NSCLC commonly develops resistance to radiation and chemotherapy, and patients are often diagnosed at stages beyond surgical remedy (5). Therefore, the exploration of novel drugs or combined chemotherapies for NSCLC treatment is urgently required.

Fisetin (3',4',7-trihydroxyflavonol), a naturally occurring flavonoid, is abundant in several fruits and vegetables, including strawberry, apple, persimmon, grape, onion and cucumber (6). At present, fisetin has shown multiple biological activities including anti-proliferative $(7,8)$, pro-apoptotic (9-13), neuroprotective (14) and anti-oxidative activities (15). Moreover, fisetin has been shown to suppress the proliferation of a wide variety of tumor cell, including prostate cancer (16), liver cancer (17), colon cancer (18) and leukemia (19) cells, and inhibit the mitogen-activated protein kinase (MAPK) and nuclear factor (NF)- $\kappa$ B signaling pathways in various type of cancer cell, such as colon and pancreatic cancer (14,20-24). Fisetin was also reported to reduce the invasive and migratory capacity of the A549 human lung cancer cell line via the extracellular signal-regulated kinase (ERK) signaling pathway (25). However, the precise impact and associated molecular mechanisms of action of fisetin in NSCLCs has remained to be fully elucidated, which was therefore the aim of the present study. The results showed that fisetin had a significant anti-tumor effect via the regulation of the proliferation, apoptosis, cell cycle and invasion of human lung cancer cells. These findings provide deep insight into the anti-tumor mechanisms of fisetin and provided a theoretical basis for its application in the clinical treatment of NSCLCs.

\section{Materials and methods}

Cell lines and culture. The A549 human NSCLC cell line was purchased from the Cell Bank of Shanghai Institutes for Biological Sciences of the Chinese Academy of Sciences (Shanghai, China) and maintained at $37^{\circ} \mathrm{C}$ in RMPI-1640 medium (Invitrogen; Thermo Fisher Scientific, Inc., Waltham, MA, USA) supplemented with $10 \%$ fetal bovine serum (FBS), $10 \mathrm{U} / \mathrm{ml}$ penicillin, $10 \mathrm{Ag} / \mathrm{ml}$ streptomycin and $0.25 \mathrm{Ag} / \mathrm{ml}$ amphotericin B (all from Gibco; Thermo Fisher Scientific, 
Inc.) in the presence of $5 \% \mathrm{CO}_{2}$. The medium was routinely changed every 2 days. Cells in the logarithmic growth phase were used for all experiments.

Drug treatment. Fisetin was purchased from Sigma-Aldrich (Merck KGaA, Darmstadt, Germany; purity, >99\% according to high-performance liquid chromatography analysis). A stock solution of fisetin was prepared in dimethyl sulfoxide (DMSO). A549 cells were seeded in 6-well plates and incubated until cells were attached to the wells. Subsequently, fisetin was added at final concentrations of 10 and $40 \mu \mathrm{M}$, followed by incubation for the indicated time periods (final DMSO concentration, $<0.2 \%$ ). Cells treated with $0.1 \%$ DMSO were used as a negative control.

Total RNA extraction and reverse-transcription quantitative polymerase chain reaction ( $R T-q P C R)$. Total RNA was isolated from A549 using TRIzol reagent (Invitrogen; Thermo Fisher Scientific, Inc.) according to the manufacturer's instructions. RNA purity and integrity were analyzed using an Agilent Bioanalyzer 2100 (Agilent Technologies, Santa Clara, CA, USA).

Complementary DNA (cDNA) was synthesized using an iScript cDNA Synthesis kit (Bio-Rad Laboratories, Inc., Hercules, CA, USA) following the manufacturer's instructions. All PCR was performed using SYBR Premix ExTaq ${ }^{\text {TII }}$ II kit (Takara, Otsu, Japan). Total RNA was degenerated at $94^{\circ} \mathrm{C}$ for $3 \mathrm{~min}$ and amplified for 40 cycles under the following conditions: $94^{\circ} \mathrm{C}$ for $5 \mathrm{sec}$ for denaturation, $52^{\circ} \mathrm{C}$ for $30 \mathrm{sec}$ for annealing and $72^{\circ} \mathrm{C}$ for $15 \mathrm{sec}$ for elongation. Detection was set at $62^{\circ} \mathrm{C}$. PCR amplifications were performed in three duplicates for each sample. The relative RNA expression was calculated using the $2^{-\Delta \Delta \mathrm{Cq}}$ method (26). The specific primer sequences are listed in Table I.

MTT assay. A549 cells were seeded at a density of $3 \times 10^{4}$ cells $/ \mathrm{ml}$ in a 24 -well plate, cultured for $24 \mathrm{~h}$ and then treated with fisetin $(0,10,20,30$ or $40 \mu \mathrm{M}$ for 24,48 or $72 \mathrm{~h})$. The cell viability was analyzed using an MTT assay according to a previously described method (27). In brief, $1 \mu 1 /$ well of MTT (Sigma-Aldrich; Merck KGaA) was added, followed by incubation at $37^{\circ} \mathrm{C}$ for an additional $4 \mathrm{~h}$. The medium was then removed and formazan was solubilized in isopropanol, followed by spectrophotometric measurement of the absorbance at $563 \mathrm{~nm}$. Each experiment was performed in triplicate and repeated three times.

Cell cycle analysis. To determine the cell cycle distribution, A549 cells treated as described above were collected, washed, suspended in PBS and fixed in 75\% ethanol. The fixed cells were stained with propidium iodide (PI) supplemented with RNaseA (Sigma-Aldrich; Merck Millipore, Darmstadt, Germany) and analyzed with a FACScan flow cytometer (BD Biosciences, Franklin Lakes, NJ, USA). Data were collected and analyzed using ModFit software 3.3 (BD Biosciences).

Flow cytometric analysis of apoptosis. For detection of apoptosis, cells were collected, washed and suspended in cold PBS for analysis. Apoptosis was detected using the Alexa Fluor ${ }^{\circledR}$
Table I. List of primers used for polymerase chain reaction.

\begin{tabular}{ll}
\hline Primer & \multicolumn{1}{c}{ Sequence (5'-3') } \\
\hline MMP-2-F & GGCCCTGTCACTCCTGAGAT \\
MMP-2-R & GGCATCCAGGTTATCGGGGA \\
MMP-9-F & AGGCCTCTACAGAGTCTTTG \\
MMP-9-R & CAGTCCAACAAGAAAGGACG \\
CDKN1A-F & CGGTGGAACTTTGACTTCGT \\
CDKN1A-R & CAGGGCAGAGGAAGTACTGG \\
CDKN1B-F & CAGAATCATAAGCCCCTGGA \\
CDKN1B-R & TCTGACGAGTCAGGCATTTG \\
CDKN2D-F & GCCTTGCAGGTCATGATGTTTGGA \\
CDKN2D-R & ATTCAGGAGCTAGGAAGCTGACCA \\
Bcl-2-F & TGCACCTGAGCGCCTTCAC \\
Bcl-2-R & TAGCTGATTCGACCATTT \\
CXCR4-F & TGACGGACAAGTACAGGCTGC \\
CXCR4-R & CCAGAAGGGAAGCGTGATGA \\
CD44-F & TATGACACATATTGCTTCAATGC \\
CD44-R & GTGTACCATCACGGTTGACA \\
E-cadherin-F & AGAACGCATTGCCACATACACTC \\
E-cadherin-R & CATTCTGATCGGTTACCGTGATC \\
GAPDH-F & CATCACCATCTTCCAGGAGCG \\
GAPDH-R & TGACCTTGCCCACAGCCTT \\
\hline
\end{tabular}

F, forward; R, reverse; MMP, matrix metalloproteinase; CDKN, cyclin D kinase inhibitor; Bcl-2, B-cell lymphoma 2; CXCR, C-X-C chemokine receptor.

647/7-AAD apoptosis kit (BioLegend, Inc., San Diego, CA, USA) according to the manufacturer's instructions. Apoptotic cells were then quantified by flow cytometry (BD Biosciences).

Cell-matrix adhesion assay. For measurement of cell adhesion, cells were pre-treated with fisetin $(0,10$ or $40 \mu \mathrm{M})$ for $24 \mathrm{~h}$ and then seeded in a 24-well plate coated with $150 \mu \mathrm{l}$ type I collagen (10 $\mu \mathrm{g} / \mathrm{ml}$; Coster, South Elgin, IL, USA) at a density of $5 \times 10^{4}$ cells $/ \mathrm{ml}$, followed by incubation for $30 \mathrm{~min}$. Non-adherent cells were then removed by PBS washes, and adherent cells were fixed in ethanol. After a staining with $0.1 \%$ crystal violet, fixed cells were lysed in $0.2 \%$ Triton X-100 and measured spectrophotometrically at $550 \mathrm{~nm}$.

Transwell invasion assay. The Transwell invasion assay was performed using the Biocoat Matrigel Invasion Chamber (BD Biosciences) according to the manufacturer's instructions. In brief, $4 \times 10^{4}$ cells were plated in the upper chambers consisting of 8-mm membrane filter inserts coated with Matrigel (BD Biosciences). The bottom chamber contained RMPI-1640 medium with $10 \%$ FBS as an inducer of invasion. After $24 \mathrm{~h}$, cells on the upper surface were removed and those attached to the lower side of the membrane were fixed and stained with crystal violet prior to counting under a microscope in five randomly selected fields.

Wound-healing assay. To determine cell motility, A549 cells $\left(1 \times 10^{5}\right.$ cells $\left./ \mathrm{ml}\right)$ were seeded in 6 -well culture plates and grown to $80-90 \%$ confluence. After aspirating the medium, 
A

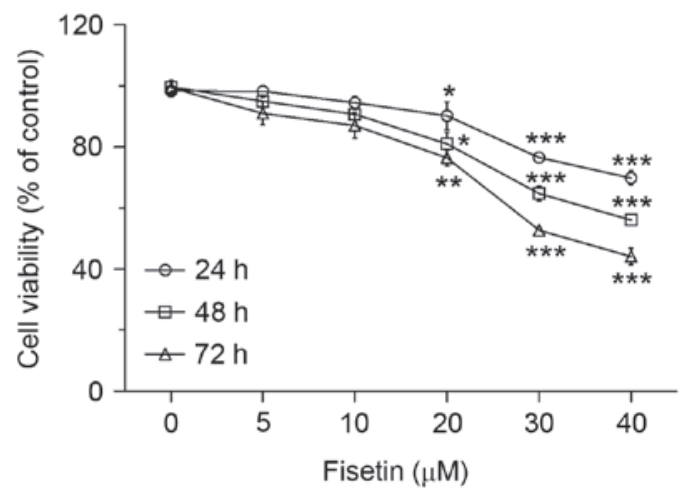

B

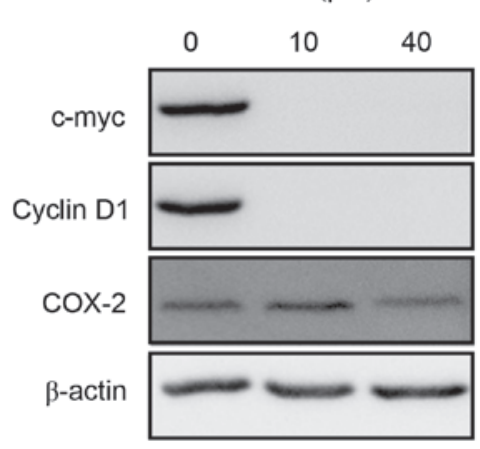

Figure 1. Effects of fisetin on the proliferation of A549 cells. A549 cells were treated with fisetin for 24,48 or $72 \mathrm{~h}$ and were then subjected to assays. (A) The cell viability was determined using the MTT assay. (B) The expression levels of the proliferation-associated genes c-myc, cyclin D1 and COX-2 in A549 cells treated with fisetin $(10$ and $40 \mu \mathrm{M})$ were detected using western blot analysis. The cells treated with $0.1 \%$ dimethylsulfoxide were used as a negative control in all these experiments. Values are expressed as the mean \pm standard deviation of at least three independent experiments. ${ }^{*} \mathrm{P}<0.05$, ${ }^{* *} \mathrm{P}<0.01,{ }^{* * *} \mathrm{P}<0.001$ as compared with control group. COX, cyclooxygenase.

the center of the cell monolayer was scraped with a sterile micropipette tip to create a denuded zone (gap) of constant width. Subsequently, cellular debris was washed with PBS and the A549 cells were then exposed to various concentrations of fisetin $(0,10$ and $40 \mu \mathrm{M})$ with serum-free medium. The wound closure was monitored and images were captured at 0 , 12, 24 and 48 h by an Olympus CKX-41 inverted microscope and an Olympus E410 camera (Olympus, Tokyo, Japan). The cells migrated across the white lines were counted in five random fields for each sample. Experiments were performed in triplicate.

Western blot analysis. Western blotting was performed as described previously (28). In brief, cells were collected and lysed with complete cell lysis (Beyotime Institute of Biotechnology, Inc., Haimen, China) with protease inhibitor cocktail (Roche, Basel, Switzerland). A total of $10 \mu$ protein was loaded and separated by $12 \%$ SDS-PAGE, transferred onto polyvinylidene difluoride membranes (Millipore, Bilerica, MA, USA) and then incubated with the appropriate antibodies specific for c-myc (\#9402; 1:2,000) Cyclin D1 (\#2922; 1:2,000), COX-2 (\#4842; 1:2,000), phosphorylated (p)-ERK1/2 (\#9101; 1:1,000), p-MAPK kinase (MEK1; \#9122; 1:1,000; all Cell Signaling Technology, Inc., Danvers, MA, USA and $\beta$-actin (1:3,000; Abcam, Cambridge, MA, USA) at $4^{\circ} \mathrm{C}$ overnight. After washing with PBS containing $0.1 \%$ Tween-20, the membranes were incubated with goat anti-rabbit IgG-horseradish peroxidase secondary antibody (sc-2004; 1:1,000; Santa Cruz Biotechnology, Inc., Dallas, TX, USA) at room temperature for $1 \mathrm{~h}$. The immunoreactive bands were visualized using an enhanced chemiluminescence system (Pierce; Thermo Fisher Scientific, Inc.) and images were captured using a CCD camera system (Tanon, Shanghai, China). The density of bands was measured by Image $\mathrm{J}$ software 2.0 (National Institutes of Health, Bethesda, MD, USA).

Caspase-3/9 fluorescent assay. Cells with appropriate treatment were collected and washed with PBS and then resuspended in lysis buffer ( $\mathrm{pH} 7.5 ; 25 \mathrm{mM}$ 4-(2-hydroxyethyl)-1-piperazineethanesulfonic acid, $5 \mathrm{~m} \mathrm{M} \mathrm{MgCl}_{2}, 5 \mathrm{mM}$ EDTA,
$5 \mathrm{mM}$ dithiothreitol, $2 \mathrm{mM}$ phenylmethane sulfonyl fluoride, $10 \mathrm{mg} / \mathrm{ml}$ pepstatin A and $10 \mathrm{mg} / \mathrm{ml}$ leupeptin). Cell lysates were centrifuged at $12,000 \mathrm{xg}$ at $4^{\circ} \mathrm{C}$ for $5 \mathrm{~min}$, and supernatants containing $50 \mathrm{mg}$ of protein were incubated with $50 \mathrm{mM}$ acetyl (Ac)-DEVD-7-amino-4-methylcoumarin (AMC; a specific substrate for caspase-3) or Ac-LEHD-AMC (a specific substrate for caspase-9; both Sigma-Aldrich; Merck Millipore) at $37^{\circ} \mathrm{C}$ for $1 \mathrm{~h}$. The fluorescence of AMC was measured using a spectrofluorometer (Hitachi F-4500; Hitachi, Tokyo, Japan) with excitation at $360 \mathrm{~nm}$ and emission at $460 \mathrm{~nm}$.

Statistical analysis. All experiments were performed independently for at least three times. Values for each group are expressed as the mean \pm standard deviation. Statistically significant differences were calculated by using a two-tailed Student's t-test with SPSS software (version 19.0; International Business Machines Corp., Armonk, NY, USA). The graphs were generated with GraphPad Prism 5.0 (GraphPad Inc., La Jolla, CA, USA).

\section{Results}

Fisetin inhibits A549 cell proliferation. The inhibitory effects of fisetin on the growth of the NSCLC cell line A549 were first assessed. Cells were treated with various doses of fisetin $(0,5,10,20,30$ or $40 \mu \mathrm{M})$ for different durations (24-72 h) and then analyzed for cell viability by an MTT assay. As expected, the results revealed that fisetin was effective in inhibiting the growth of A549 cells in a dose- and time-dependent manner (Fig. 1A). According to the growth curves, fisetin concentrations of 10 and $40 \mu \mathrm{M}$ were used in the subsequent mechanistic experiments. The protein expression levels of the proliferation-associated genes, cyclin D1, c-myc and cyclooxygenase (COX)-2, were also assessed; the three genes were downregulated by fisetin (Fig. 1B).

Fisetin causes cell cycle arrest in A549 cells. Due to the anti-proliferative effects of fisetin on A549 cells, it was further investigated whether the growth inhibitory effect of fisetin was mediated through cell cycle arrest. For this purpose, the 

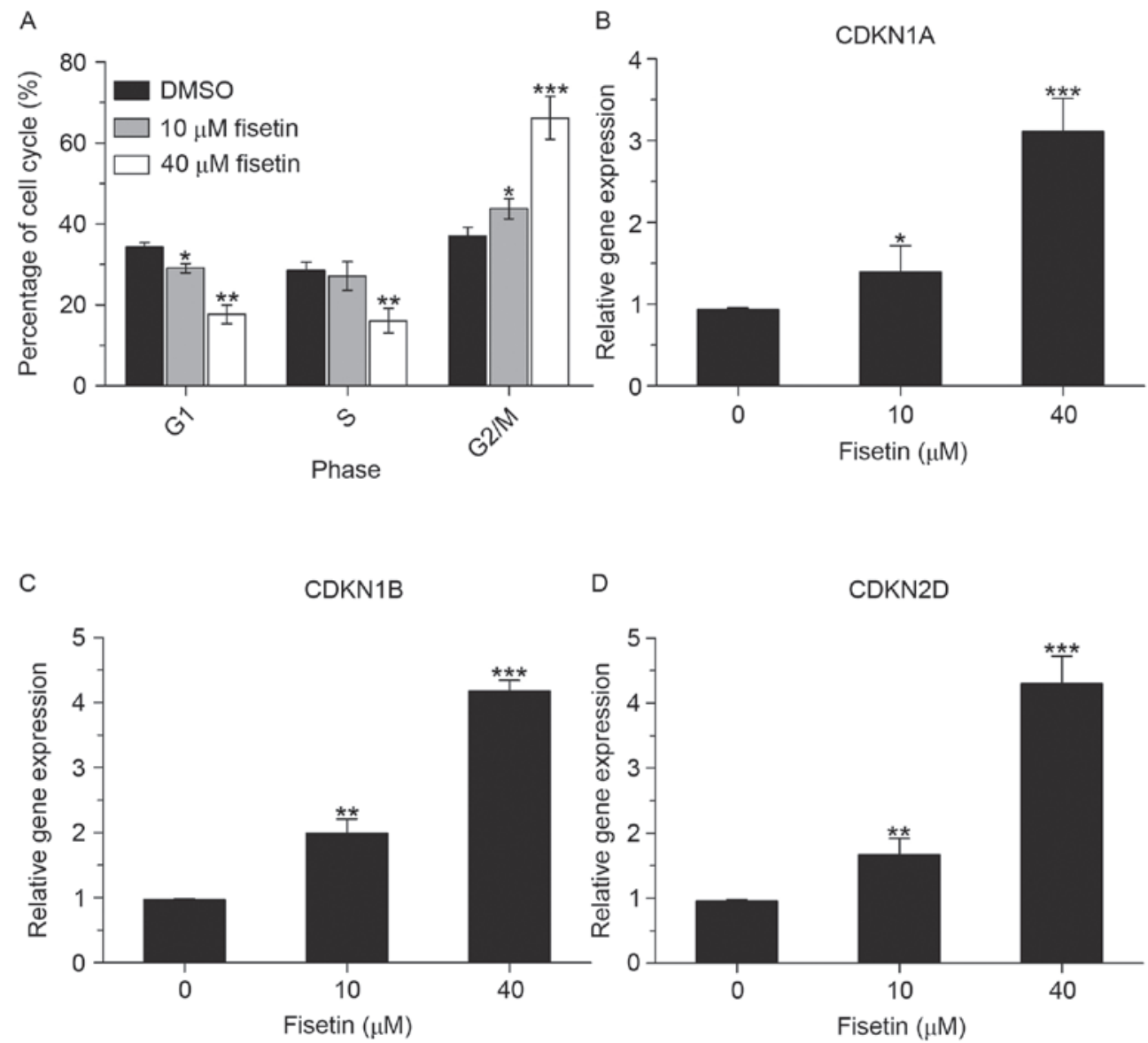

Figure 2. Effects of fisetin on the cell cycle of A549 cells. (A) A549 cells treated with fisetin were collected, stained with propidium iodide and the cell cycle distribution was analyzed with a flow cytometer. (B-D) The expression levels of cell cycle-associated genes (B) CDKN1A, (C) CDKN1B and (D) CDKN2D in A549 cells were analyzed by reverse-transcription quantitative polymerase chain reaction assays. Cells treated with DMSO were adopted as a negative control. Values are expressed as the mean \pm standard deviation of at least three independent experiments. ${ }^{*} \mathrm{P}<0.05,{ }^{* * *} \mathrm{P}<0.01$ and ${ }^{* * * *} \mathrm{P}<0.001$ as compared with control group. DMSO, dimethyl sulfoxide; CDKN, cyclin D kinase inhibitor.

effect of fisetin on cell cycle alterations in A549 cells was measured by flow cytometry. As shown in Fig. 2A, fisetin caused an accumulation of cells in G2/M phase and a reduction in G1 phase of the cell cycle. In the $40 \mu \mathrm{M}$ fisetin-treated group, the percentage of cells in G1 phase decreased from 34.3 to $17.6 \%$ and the percentage of cells in $\mathrm{G} 2 / \mathrm{M}$ phase increased from 37.1 to $66.2 \%$. Furthermore, the mRNA expression levels of cell cycle-associated genes cyclin D kinase inhibitor (CDKN) 1A, CDKN1B and CDKN2D were significantly upregulated (Fig. 2B-D). This finding indicated that fisetin induced cell cycle arrest in $\mathrm{G} 2$ phase by regulating the expression of multiple cell cycle regulatory genes.

Fisetin induces apoptosis of A549 cells. To determine whether blockage of cells in G2/M phase by fisetin induces apoptosis of A549 cells, flow cytometric analysis, using an Alexa Fluor 647/7-AAD apoptosis kit, was performed. A549 cells were treated with 10 and $40 \mu \mathrm{M}$ fisetin for 6,12 and $24 \mathrm{~h}$, and then collected for flow cytometric analysis. As shown in Fig. 3A, the apoptotic rate of A549 cells was markedly increased by fisetin. The mRNA expression levels of anti-apoptotic genes (B-cell lymphoma-2, C-X-C chemokine receptor 4 , CD44 and E-cadherin) were all downregulated, which was consistent with the increases in the apoptotic rate (Fig. 3B-E).
Moreover, the effects of fisetin treatment on the activation of caspase-3 and -9 , which have crucial roles in apoptosis, were further investigated. Caspase fluorescence assays were used to evaluate caspase- 3 and caspase- 9 activity, and the results showed that the activation of caspase- 3 and caspase- 9 was obviously increased by fisetin (Fig. 3F).

Fisetin suppresses cell adhesion, invasion and migration. The potential anti-metastatic activity of fisetin in A549 cells was then investigated. A549 cells were treated with 10 or $40 \mu \mathrm{M}$ fisetin and cell adhesion, invasion and migration were assessed. As expected, a cell-matrix adhesion assay revealed that $40 \mu \mathrm{M}$ fisetin had a significant inhibitory effect on cell adhesion, reducing it to $35.4 \%$ of that of the untreated control (Fig. 4A). A Transwell migration assay showed that the invasiveness of A549 cells treated with fisetin was suppressed compared with that of the negative control cells (Fig. 4B). In addition, a wound healing assay showed that the motility of A549 cells treated with fisetin was suppressed compared with that of the negative control cells (Fig. 4C).

The present study further examined the mRNA expression levels of invasion-associated genes. The results were consistent with the effect of fisetin on the adhesion, migration and invasion of A549 cells, as the mRNA expression of matrix 
A

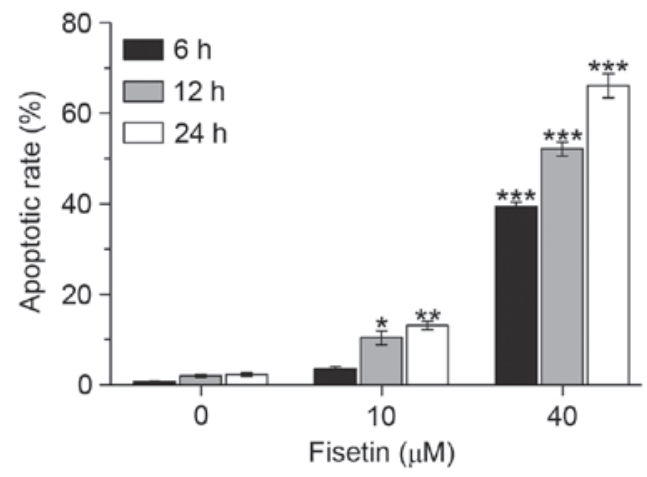

C

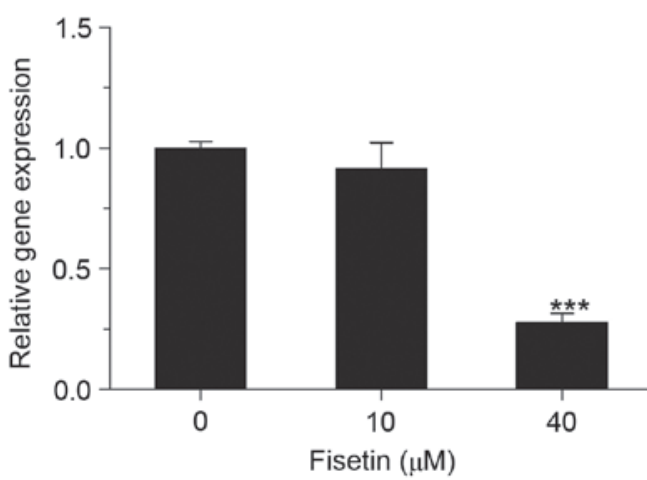

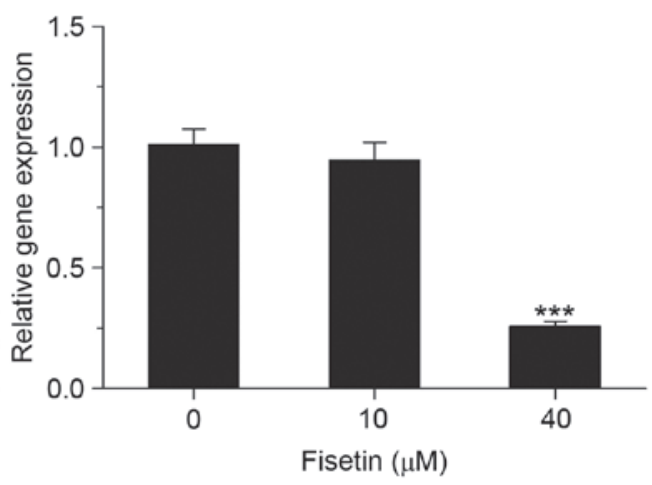

D

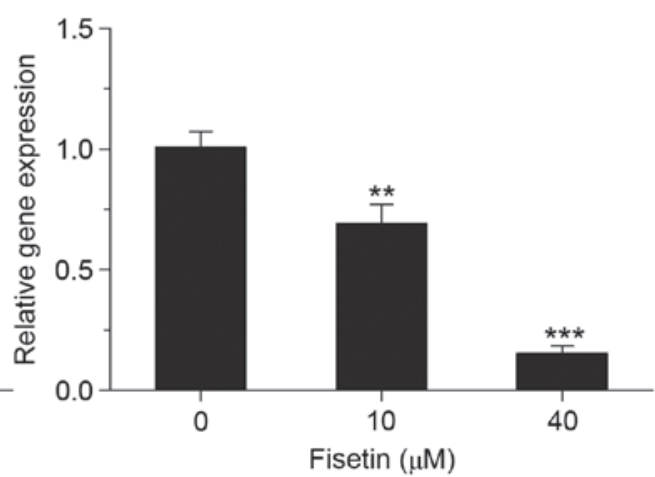

E

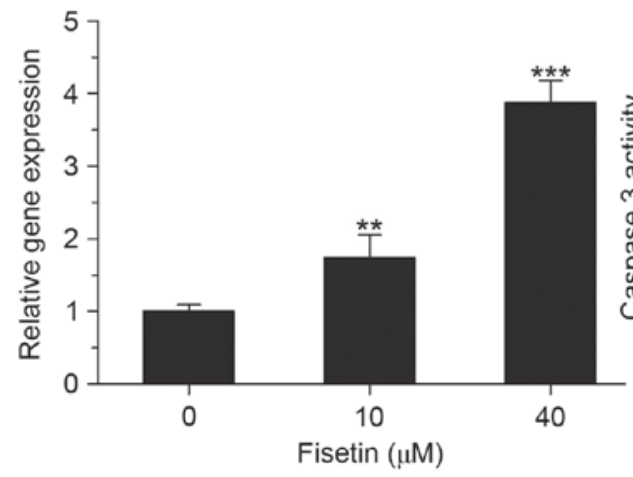

$\mathrm{F}$

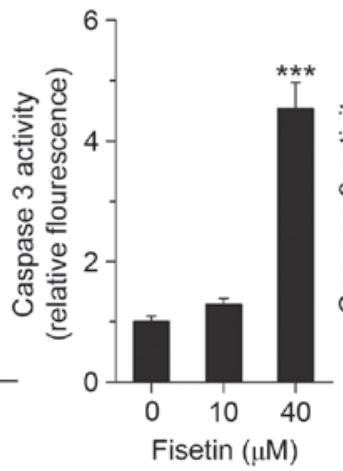

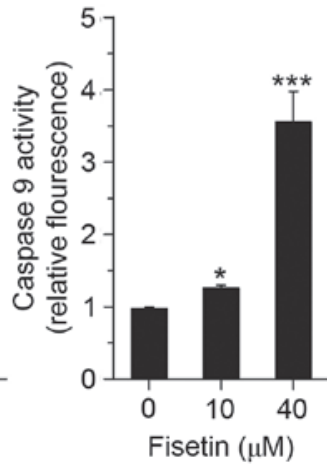

Figure 3. Effects of fisetin on apoptosis of A549 cells. (A) A549 cells treated with fisetin for 6-24 h and the apoptotic rate was examined using flow cytometric analysis. (B-E) The expression levels of apoptosis-associated genes (B) Bcl-2, (C) CXCR4, (D) CD44 and (E) E-cadherin in A549 cells were analyzed by reverse-transcription quantitative polymerase chain reaction assays. (F) The activation of caspase 3 and caspase 9 was determined using a caspase-3/9 fluorescent assay. Values are expressed as the mean \pm standard deviation of at least three independent experiments. ${ }^{*} \mathrm{P}<0.05,{ }^{* * *} \mathrm{P}<0.01,{ }^{* * * *} \mathrm{P}<0.001$ as compared with control group. Bcl-2, B-cell lymphoma 2; CXCR, C-X-C chemokine receptor.

metalloproteinase (MMP)-2 and MMP-9 was suppressed after fisetin treatment. It is therefore suggested that fisetin inhibits the adhesion, invasion and migration of lung cancer cells via regulating the expression of associated genes, such as MMP-2 and MMP-9 (Fig. 4D and E).

Effect of fisetin on the MAPK signaling pathway. Since ERK1/2-associated signaling has a crucial role in cancer biology and fisetin has been reported to inhibit melanoma cell invasion by targeting the MAPK signaling pathway (29), the present study further investigated its effect in lung cancer cells. First, the phosphorylation status of ERK1/2 was determined. Western blot assays showed that p-ERK1/2 was markedly downregulated after fisetin treatment at the low as well as the high dose (10 and $40 \mu \mathrm{M})$. Since activation of the ERK pathway is regulated by MEK1, its activity of MEK1 was also assessed. The results were similar to those on ERK, as the level of p-MEK1 was decreased in the fisetin treatment group, which suggested that fisetin inhibits the activation of the ERK signaling pathway via MEK1/2 to reduce the growth and invasion in A549 cells (Fig. 4F).

\section{Discussion}

Non-small cell lung cancer, the most common type of cancer, remains a huge challenge to the public health sector. Current 
A

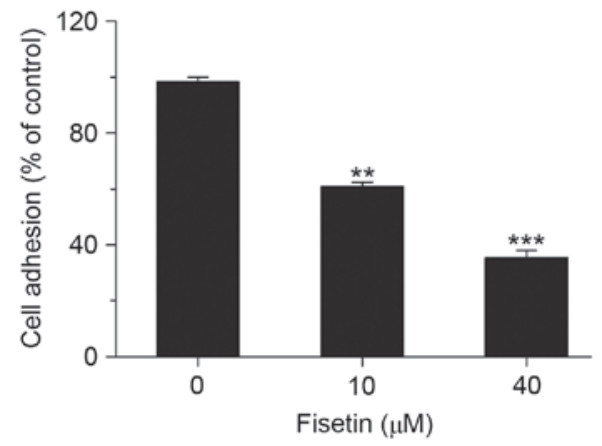

C

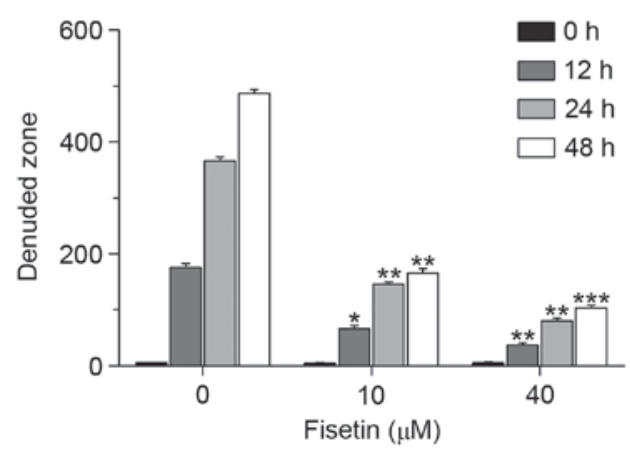

$\mathrm{E}$

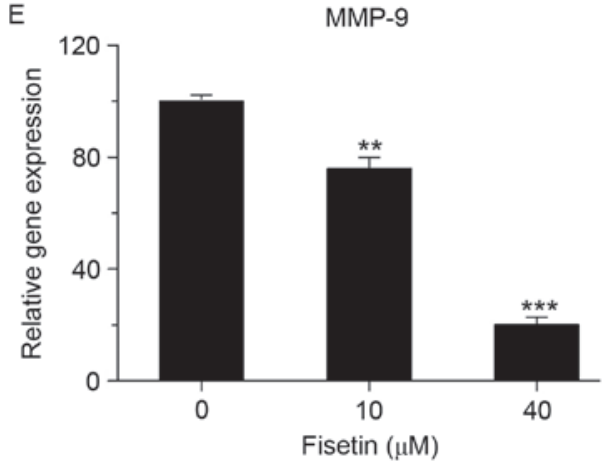

B

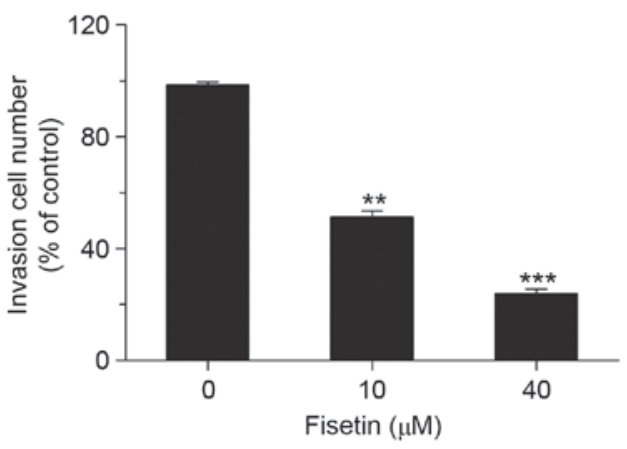

D

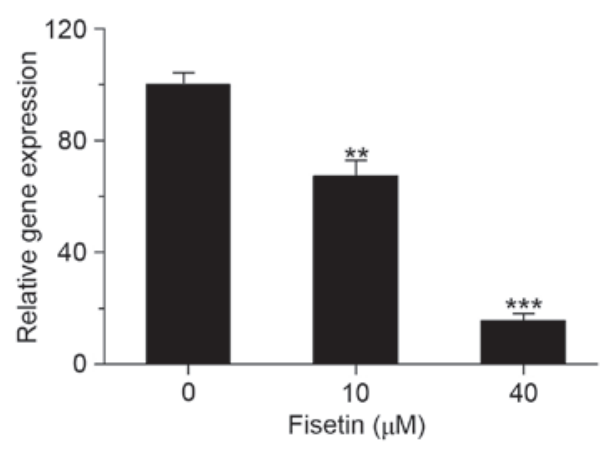

F

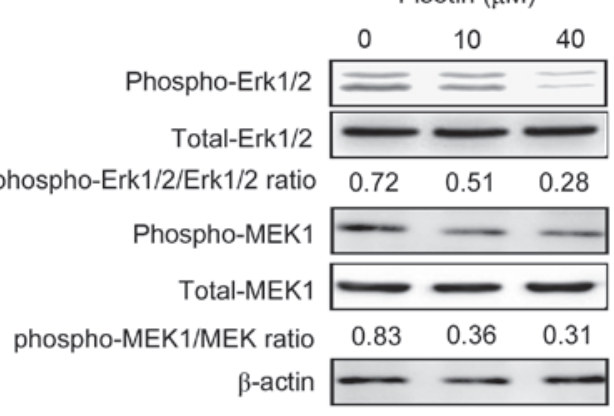

Figure 4. Effects of fisetin on cell adhesion, invasion and migration of A549 cells. (A) Cells were treated with various concentrations $(0,10$ or $40 \mu \mathrm{M})$ of fisetin for $24 \mathrm{~h}$, and were then subjected to analyses for cell-matrix adhesion. (B) The invasiveness of A549 cells treated with fisetin was measured by a Transwell assay. (C) The migration of A549 cells treated with fisetin was measured by a wound-healing assay. (D and E) Changes in the expression levels of cell invasion-associated genes (D) MMP-2 and (E) MMP-9 in A549 cells was analyzed by reverse-transcription quantitative polymerase chain reaction assays. (F) The phosphorylation status of ERK1/2 and MEK1 of A549 cells treated with fisetin was examined by western blot analysis. Values are expressed as the mean \pm standard deviation of at least three independent experiments. ${ }^{*} \mathrm{P}<0.05,{ }^{* *} \mathrm{P}<0.01,{ }^{* * *} \mathrm{P}<0.001$ as compared with control group. Phospho-ERK1/2, phosphorylated extracellular signal-regulated kinase 1/2; MEK1, mitogen-activated protein kinase kinase.

treatments include surgical resection and chemotherapy, which is limited by toxicity and side effects. Therefore, a growing number of studies are aiming to find more effective anti-tumor drugs, which may prevent carcinogenesis, curtail its progression or even cure the disease.

Fisetin, a natural polyphenol abundantly found in several fruits and vegetables, has attracted increasing attention for its anti-tumor effect in certain types of cancer cell. In the present study, the non-toxic dietary flavonoid fisetin was found to greatly inhibit the growth of NSCLC cells in vitro via inhibiting cell proliferation, inducing cell-cycle arrest in G2 phase, suppressing cell invasion and migration, and promoting cell apoptosis.

The MAPK pathways serve to coordinate key cellular processes. The ERK1/2 pathway has key roles in the regulation of multiple biological activities and constitutive activation of the ERK1/2 pathway contributes to tumorigenesis or cancer growth, and increases the cell death threshold (30). ERK1/2 may be activated by MEK1 (31). The results of the present study indicated that fisetin suppresses cell invasion by predominantly targeting the MAPK signaling pathway as the activation of ERK1/2 was markedly downregulated by fisetin at a high and low dose, which is consistent with previous results (32). Accordingly, it is suggested that fisetin suppresses NSCLCs cell growth via the ERK1/2 pathway.

Collectively, all of these results suggested that fisetin effectively inhibited cell proliferation and migration, and induced apoptosis in NSCLCs. The present study demonstrated the potential anti-cancer effects of fisetin in NSCLCs and the 
results may provide a theoretical basis to support the application of fisetin in the clinical treatment of lung cancer.

\section{References}

1. Ferlay J, Soerjomataram I, Dikshit R, Eser S, Mathers C, Rebelo M, Parkin DM, Forman D and Bray F: Cancer incidence and mortality worldwide: Sources, methods and major patterns in GLOBOCAN 2012. Int J Cancer 136: E359-E386, 2015.

2. Sullivan I and Planchard D: ALK inhibitors in non-small cell lung cancer: The latest evidence and developments. Ther Adv Med Oncol 8: 32-47, 2016.

3. Luque M, Díez FJ and Disdier C: Optimal sequence of tests for the mediastinal staging of non-small cell lung cancer. BMC Med Inform Decis Mak 16: 9, 2016

4. Kawasaki K, Sato Y, Suzuki Y, Saito H, Nomura Y and Yoshida Y: Prognostic factors for surgically resected N2 non-small cell lung cancer. Ann Thorac Cardiovasc Surg 21: 217-222, 2015.

5. Hsu YL, Kuo PL and Lin CC: Proliferative inhibition, cell-cycle dysregulation, and induction of apoptosis by ursolic acid in human non-small cell lung cancer A549 cells. Life Sci 75: 2303-2316, 2004.

6. Arai Y, Watanabe S, Kimira M, Shimoi K, Mochizuki R and Kinae N: Dietary intakes of flavonols, flavones and isoflavones by Japanese women and the inverse correlation between quercetin intake and plasma LDL cholesterol concentration. J Nutr 130 : 2243-2250, 2000.

7. Haddad AQ, Venkateswaran V, Viswanathan L, Teahan SJ, Fleshner NE and Klotz LH: Novel antiproliferative flavonoids induce cell cycle arrest in human prostate cancer cell lines. Prostate Cancer Prostatic Dis 9: 68-76, 2006.

8. Suh Y, Afaq F, Khan N, Johnson JJ, Khusro FH and Mukhtar H: Fisetin induces autophagic cell death through suppression of mTOR signaling pathway in prostate cancer cells. Carcinogenesis 31 : 1424-1433, 2010.

9. Pal HC, Sharma S, Elmets CA, Athar M and Afaq F: Fisetin inhibits growth, induces G2/M arrest and apoptosis of human epidermoid carcinoma A431 cells: Role of mitochondrial membrane potential disruption and consequent caspases activation. Exp Dermatol 22: 470-475, 2013.

10. Suh Y, Afaq F, Johnson JJ and Mukhtar H: A plant flavonoid fisetin induces apoptosis in colon cancer cells by inhibition of COX2 and Wnt/EGFR/NF-kappaB-signaling pathways. Carcinogenesis 30: 300-307, 2009.

11. Khan N, Asim M, Afaq F, Abu Zaid M and Mukhtar H: A novel dietary flavonoid fisetin inhibits androgen receptor signaling and tumor growth in athymic nude mice. Cancer Res 68: 8555-8563, 2008.

12. Khan N, Afaq F, Syed DN and Mukhtar H: Fisetin, a novel dietary flavonoid, causes apoptosis and cell cycle arrest in human prostate cancer LNCaP cells. Carcinogenesis 29: 1049-1056, 2008.

13. Chen YC, Shen SC, Lee WR, Lin HY, Ko CH, Shih CM and Yang LL: Wogonin and fisetin induction of apoptosis through activation of caspase 3 cascade and alternative expression of p21 protein in hepatocellular carcinoma cells SK-HEP-1. Arch Toxicol 76: 351-359, 2002.

14. Maher P, Akaishi T and Abe K: Flavonoid fisetin promotes ERK-dependent long-term potentiation and enhances memory. Proc Natl Acad Sci USA 103: 16568-16573, 2006.

15. Hou DX, Fukuda M, Johnson JA, Miyamori K, Ushikai M and Fujii M: Fisetin induces transcription of NADPH: Quinone oxidoreductase gene through an antioxidant responsive element-involved activation. Int J Oncol 18: 1175-1179, 2001

16. Haddad AQ, Venkateswaran V, Viswanathan L, Teahan SJ, Fleshner NE and Klotz LH: Novel antiproliferative flavonoids induce cell cycle arrest in human prostate cancer cell lines. Prostate Cancer Prostatic Dis 9: 68-76, 2006.
17. Chen YC, Shen SC, Lee WR, Lin HY, Ko CH, Shih CM and Yang LL: Wogonin and fisetin induction of apoptosis through activation of caspase 3 cascade and alternative expression of p21 protein in hepatocellular carcinoma cells SK-HEP-1. Arch Toxicol 76: 351-359, 2002.

18. Lu H, Chang DJ, Baratte B, Meijer L and Schulze-Gahmen U: Crystal structure of a human cyclin-dependent kinase 6 complex with a flavonol inhibitor, fisetin. J Med Chem 48: 737-743, 2005.

19. Lee WR, Shen SC, Lin HY, Hou WC, Yang LL and Chen YC: Wogonin and fisetin induce apoptosis in human promyeloleukemic cells, accompanied by a decrease of reactive oxygen species, and activation of caspase 3 and $\mathrm{Ca}(2+)$-dependent endonuclease. Biochem Pharmacol 63: 225-236, 2002.

20. Yao K, Zhang L, Zhang Y, Ye P and Zhu N: The flavonoid, fisetin, inhibits UV radiation-induced oxidative stress and the activation of NF-kappaB and MAPK signaling in human lens epithelial cells. Mol Vis 14: 1865-1871, 2008

21. Murtaza I, Adhami VM, Hafeez BB, Saleem M and Mukhtar H: Fisetin, a natural flavonoid, targets chemoresistant human pancreatic cancer AsPC-1 cells through DR3-mediated inhibition of NF-kappaB. Int J Cancer 125: 2465-2473, 2009.

22. Kim SC, Kang SH, Jeong SJ, Kim SH, Ko HS and Kim SH: Inhibition of c-Jun N-terminal kinase and nuclear factor $\kappa \mathrm{B}$ pathways mediates fisetin-exerted anti-inflammatory activity in lipopolysccharide-treated RAW264. 7 cells. Immunopharmacol Immunotoxicol 34: 645-650, 2012

23. Goh FY, Upton N, Guan S, Cheng C, Shanmugam MK, Sethi G, Leung BP and Wong WF: Fisetin, a bioactive flavonol, attenuates allergic airway inflammation through negative regulation of NF-кB. Eur J Pharmacol 679: 109-116, 2012.

24. Léotoing L, Wauquier F, Guicheux J, Miot-Noirault E, Wittrant Y and Coxam V: The polyphenol fisetin protects bone by repressing $\mathrm{NF}-\kappa \mathrm{B}$ and MKP-1-dependent signaling pathways in osteoclasts. PLoS One 8: e68388, 2013

25. Liao YC, Shih YW, Chao CH, Lee XY and Chiang TA Involvement of the ERK signaling pathway in fisetin reduces invasion and migration in the human lung cancer cell line A549. J Agric Food Chem 57: 8933-8941, 2009.

26. Livak KJ and Schmittgen TD: Analysis of relative gene expression data using real-time quantitative PCR and the 2(-Delta Delta C(T)) Method. Methods 25: 402-408, 2001.

27. Lin R, Maeda S, Liu C, Karin M and Edgington TS: A large noncoding RNA is a marker for murine hepatocellular carcinomas and a spectrum of human carcinomas. Oncogene 26: 851-858, 2007.

28. Li S, Dong P, Wang J, Zhang J, Gu J, Wu X, Wu W, Fei X, Zhang Z, Wang Y, et al: Icariin, a natural flavonol glycoside, induces apoptosis in human hepatoma SMMC-7721 cells via a ROS/JNK-dependent mitochondrial pathway. Cancer Lett 298: 222-230, 2010

29. Pal HC, Sharma S, Strickland LR, Katiyar SK, Ballestas ME, Athar M, Elmets CA and Afaq F: Fisetin inhibits human melanoma cell invasion through promotion of mesenchymal to epithelial transition and by targeting MAPK and NFאB signaling pathways. PLoS One 9: e86338, 2014.

30. Ishikawa Y and Kitamura M: Dual potential of extracellular signal-regulated kinase for the control of cell survival. Biochem Biophys Res Commun 264: 696-701, 1999.

31. Ellinger-Ziegelbauer H, Brown K, Kelly K and Siebenlist U: Direct activation of the stress-activated protein kinase (SAPK) and extracellular signal-regulated protein kinase (ERK) pathways by an inducible mitogen-activated protein Kinase/ERK kinase kinase 3 (MEKK) derivative. J Biol Chem 272: 2668-2674, 1997.

32. Chou RH, Hsieh SC, Yu YL, Huang MH, Huang YC and Hsieh YH: Fisetin inhibits migration and invasion of human cervical cancer cells by down-regulating urokinase plasminogen activator expression through suppressing the p38 MAPK-dependent NF- $\kappa$ B signaling pathway. PLoS One 8: e71983, 2013 